\title{
Electrochemical Capillary-Flow Immunoassay for Detecting Anti- SARS-CoV-2 Nucleocapsid Protein Antibodies at the Point of Care
}

\author{
Isabelle C. Samper, Ana Sánchez-Cano, Wisarut Khamcharoen, Ilhoon Jang, Weena Siangproh, \\ Eva Baldrich, Brian J. Geiss, David S. Dandy, and Charles S. Henry*
}

Cite This: https://doi.org/10.1021/acssensors.1c01527

Read Online

\section{ACCESS |}

山ll Metrics \& More

回国 Article Recommendations

Supporting Information

ABSTRACT: Rapid and inexpensive serological tests for SARS-CoV-2 antibodies are needed to conduct population-level seroprevalence surveillance studies and can improve diagnostic reliability when used in combination with viral tests. Here, we report a novel low-cost electrochemical capillary-flow device to quantify IgG antibodies targeting SARS-CoV-2 nucleocapsid proteins (anti-N antibody) down to $5 \mathrm{ng} / \mathrm{mL}$ in low-volume $(10 \mu \mathrm{L})$ human whole blood samples in under $20 \mathrm{~min}$. No sample preparation is needed as the device integrates a blood-filtration membrane for on-board plasma extraction. The device is made of stacked layers of a hydrophilic polyester and double-sided adhesive films, which create a passive microfluidic circuit that automates the steps of an enzyme-linked immunosorbent assay (ELISA). The sample and reagents are sequentially delivered to a nitrocellulose membrane that is modified

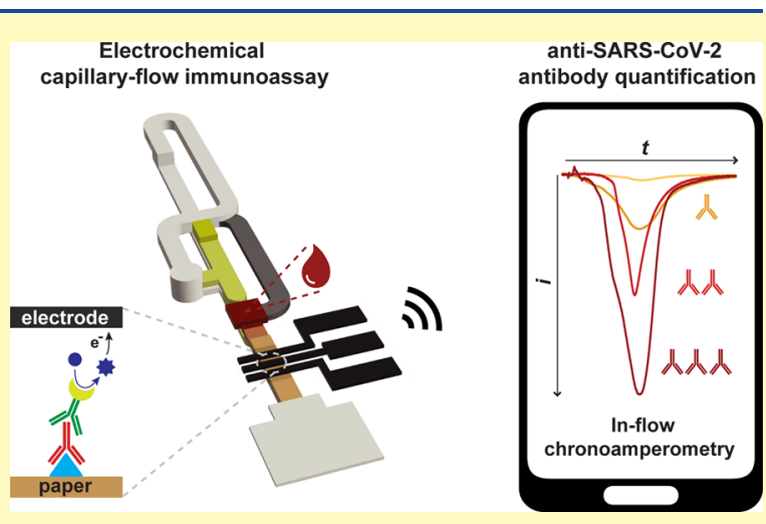
with a recombinant SARS-CoV-2 nucleocapsid protein. When present in the sample, anti- $\mathrm{N}$ antibodies are captured on the nitrocellulose membrane and detected via chronoamperometry performed on a screen-printed carbon electrode. As a result of this quantitative electrochemical readout, no result interpretation is required, making the device ideal for point-of-care (POC) use by non-trained users. Moreover, we show that the device can be coupled to a near-field communication potentiostat operated from a smartphone, confirming its true POC potential. The novelty of this work resides in the integration of sensitive electrochemical detection with capillary-flow immunoassay, providing accuracy at the point of care. This novel electrochemical capillary-flow device has the potential to aid the diagnosis of infectious diseases at the point of care.

KEYWORDS: point-of-care (POC) diagnostics, capillary-flow device, electrochemistry, SARS-CoV-2, serology

\section{INTRODUCTION}

Coronavirus disease (COVID-19) caused by the severe acute respiratory syndrome coronavirus 2 (SARS-CoV-2) was classified as a pandemic by the World Health Organization and has caused over $4 \mathrm{M}$ deaths worldwide as of July $2021 .^{1}$ In order to contain the pandemic, different strategies have been developed within the last year to detect SARS-CoV-2 infections and monitor the virus spread. Both antigen and antibody detection play important roles in pandemic management as well as assessment of patient prognosis.

Reverse transcription polymerase chain reaction (RT-qPCR) is considered to be the gold standard method for detecting the presence of virus, while rapid antigen tests are used for screening at point-of-care (POC) facilities. ${ }^{2}$ Meanwhile, serological testing has become key for population-level surveillance because it can be used to detect previous exposure to SARS-CoV-2, even in asymptomatic patients, and enables identification of individuals that remain susceptible to the virus. ${ }^{3}$ Thus, seroprevalence analysis can guide decision making around social restrictions and lockdown measures that are in place in most countries. ${ }^{4}$ Moreover, serological assays can be used in combination with RT-qPCR to improve COVID-19 diagnostic reliability, ${ }^{5,6}$ as well as to establish contact tracing, to identify convalescence plasma donors and to study antibody titers following vaccination.

Current serological assays for COVID-19 include neutralization assays, enzyme-linked immunosorbent assays (ELISAs), and chemiluminescent immunoassays. ${ }^{8}$ Whilst being highly sensitive, these assays take hours to days to provide results and require laboratory instrumentation that is not widely available in low-income settings or at the point of care. ${ }^{9}$ In response to the need for rapid tests imposed by the worldwide pandemic, multiple COVID-19 serology assays, ${ }^{6,8,10,11}$ including POC assays $^{12}$ are rapidly emerging. Of the rapid COVID-19 serology tests that are commercially available, most use

Received: July 19, 2021

Accepted: October 12, 2021 
lateral-flow immunoassay (LFIA) formats. While LFIAs offer a simple operation and deliver fast results, ${ }^{13}$ their clinical sensitivity remain far lower than standard laboratory methods, limiting their impact on pandemic control and management. ${ }^{14}$ In addition, LFIAs require an advanced reader to give quantitative results.

Electrochemical bioanalytical methods have gained considerable interest in recent years ${ }^{15}$ because they provide easy and rapid signal quantification, require simple instrumentation, and enable assay miniaturization. ${ }^{16}$ Critically, sensitive electrodes can be integrated in microsystems, enabling the analysis of small-volume samples. ${ }^{17}$ Coupled with immunoassays, they can achieve extremely low detection limits. ${ }^{18,19}$ Electrochemical immunoassays have been successfully applied to the detection of viral antigens $s^{20,21}$ and anti-viral antibodies. ${ }^{22,23}$ While providing great analytical sensitivity and clinically relevant dynamic range, electrochemical immunoassays typically require multistep procedures and laboratory-based equipment. ${ }^{18,19}$ Yet, with the growing use of carbon as an electrode material and the miniaturization of electronic instrumentation, electrochemical assays can now be made low-cost and portable, allowing for high-performance diagnostic assays to be deployable at the point of care. ${ }^{24-28}$

Electrochemical paper-based devices (ePADs) have attracted widespread attention in biosensing due to their numerous advantages. $^{29}$ A paper is one of the most common materials used in capillary-flow devices because it is affordable, flexible, easy to dispose, and allows simple device fabrication. ${ }^{30}$ ePADs are used to transport solutions through microfluidic channels, delivering them to an electrode that drives an electrochemical reaction. The flow is driven by the capillary force of the paper, without the need for an external pump. As a result, ePADs provide portability and simplicity while achieving high analytical performance offered by electrochemical techniques. $^{31}$ However, difficulty with controlling flow and the resulting non-uniform flow rates are the known limitations of ePADs. Capillary-driven microfluidic systems, such as laminated devices made of polyester films, were developed to overcome these limitations, enabling uniform, fast, and accurate flow control. ${ }^{32,33}$ Compared to paper-based devices, which suffer from nonspecific adsorption of reagents and analytes on the cellulose fibers, laminated devices are less adsorbent, hence limiting this problem. Due to their improved flow capacities and limited nonspecific adsorption, laminated devices offer better analytical performance than paper-based devices. $^{33}$

Here, we present an electrochemical capillary-flow immunoassay that combines the ease of use of an LFIA with the high sensitivity of electrochemical detection methods. Our novel assay is applied to the detection of $\operatorname{IgG}$ antibodies against a SARS-CoV-2 nucleocapsid protein (anti-N antibodies) in human blood samples. An anti-N IgG antibody was chosen as the analytical target of our novel device because of its clinical relevance in COVID-19 diagnosis and patient prognosis. $^{34-36}$ Based on our previous work, ${ }^{33,37}$ the device developed here is made of stacked layers of polyester and double-sided adhesive films, which create a capillary-flow microfluidic circuit. The device integrates a blood-filtration membrane for on-board plasma extraction. Dried reagents along the microfluidic flow path are rehydrated during device operation and sequentially delivered to a detection zone. The detection zone consists of a stencil-printed carbon electrode (SPCE) held onto a nitrocellulose membrane (NCM) that is modified with capturing agents specific to the target analyte, anti-N antibody. If present in the sample, anti-N antibody is detected using chronoamperometry in a sandwich assay setup. Development of the electrochemical capillary-flow device is presented, and a sensor response is characterized. As a proofof-concept experiment, the device is coupled to a near-field communication (NFC) potentiostat operated from a smartphone, demonstrating its full potential for POC use.

\section{EXPERIMENTAL SECTION}

Reagent Preparation. $N$ Protein. A recombinant SARS-CoV-2 nucleocapsid protein ( $\mathrm{N}$ protein) was produced as described elsewhere. $^{38,39}$ Briefly, a bacteria-codon optimized gBlock was cloned into a pET28a bacterial expression with a C-terminal 6xHis tag. A recombinant protein was expressed in BL21(DE3) pLysS E. coli and purified by nickel affinity and size exclusion chromatography. To minimize aggregation, $50 \mathrm{mM}$ of HEPES buffer ( $\mathrm{pH} 7.4$ ), $500 \mathrm{mM}$ of $\mathrm{NaCl}$ was used throughout the purification process. $\mathrm{N}$ protein purity was assessed using sodium dodecyl sulfate-polyacrylamide gel electrophoresis. A solution of $0.5 \mathrm{mg} / \mathrm{mL}$ recombinant $\mathrm{N}$ protein was prepared in $45 \mathrm{mM}$ of trehalose and $4.5 \%$ glycerol and striped onto a $3 \times 16 \mathrm{~mm} \mathrm{NCM} \mathrm{(FF120,} \mathrm{GE)} \mathrm{using} \mathrm{a} \mathrm{reagent} \mathrm{dispenser}$ (Claremont Bio), so that $120 \mathrm{ng} \mathrm{N}$ protein was dispensed on each strip. $\mathrm{N}$ protein striped $\mathrm{NCMs}$ were stored at $4{ }^{\circ} \mathrm{C}$ for up to a month before use, as stability over storage was illustrated elsewhere. ${ }^{37}$

HRP-Antibody. Anti-mouse-IgG conjugated to horseradish peroxidase (HRP-antibody; ab97040, Abcam) was used as a detection antibody. A solution of $5 \mu \mathrm{g} / \mathrm{mL}$ HRP-antibody was prepared in 0.01 $\mathrm{M} \mathrm{FeSO}_{4}-$ EDTA, $4 \%$ trehalose, and $0.1 \%$ BSA to enable storage of the dried antibodies for at least 5 months. ${ }^{40} \mathrm{HRP}$-antibody reagent pads were made by pipetting $10 \mu \mathrm{L}$ of this solution onto $3 \times 5 \mathrm{~mm}$ glass fiber pads (Millipore Sigma, GXDX203000) and were then dried at $37^{\circ} \mathrm{C}$ for $30 \mathrm{~min}$. They were stored at $4{ }^{\circ} \mathrm{C}$ for up to a week before use.

Anti-N Antibody. Antibodies against SARS-CoV-2 N proteins (GTX632269, GeneTex Inc.) were spiked in $0.1 \mathrm{M}$ phosphate buffer saline (PBS) pH 7.4 (PBS-based sample) or in blood samples. Whole human blood from healthy individuals was obtained from Lee Biosolutions.

Other Reagents. The electrochemical substrate used throughout this study was 3,3',5,5' -tetramethylbenzidine (TMB), purchased from Sigma-Aldrich (T0440). The washing buffer solution consisted of 0.1 M PBS ( $\mathrm{pH} 7.4)$ and $0.1 \%$ Tween 80 . All solutions were prepared using ultrapure water purified using a Milli-Q system (Millipore Sigma).

Electrode Fabrication. Electrodes were fabricated using a stencilprinting method described previously. ${ }^{41}$ Graphite powder (3569, Asbury Carbons) was hand-mixed with carbon ink (E3178, Ercon Inc.) in the ratio $0.6-1(\% \mathrm{w} / \mathrm{w})$ to create a graphite paste. Single-use stencils were made of $100 \mu \mathrm{m}$ thick polyester films (PP2500, $3 \mathrm{M}$ ), which were cut using a $\mathrm{CO} 2$ laser cutter (Zing 1000, Epilog) following a pattern designed with CorelDRAW. A stencil was taped onto a whole polyester film (PP2500, $3 \mathrm{M}$ ) and a squeegee was used to print the graphite paste onto the film. Upon removal of the stencil, the SPCEs were cured at $60{ }^{\circ} \mathrm{C}$ for $30 \mathrm{~min}$. SPCEs were then plasma treated at $500 \mathrm{mTorr}, 125 \mathrm{~W}$ for $2 \mathrm{~min}$ to improve electrochemical performance. ${ }^{41}$ Finally, AglAgCl ink (901773, Sigma-Aldrich) was hand-painted on the reference electrode (RE) and cured at $60{ }^{\circ} \mathrm{C}$ for $30 \mathrm{~min}$. Plasma-treated electrodes, as fabricated here, have been shown to be stable for at least 10 days $^{41}$ and were used for up to a week following plasma treatment.

Capillary-Flow Device Fabrication. The capillary-flow device was fabricated similarly to a device previously reported, ${ }^{37}$ with major differences including the design and number of layers, the materials used and the integrated SPCE. Here, the device was constructed by stacking four layers of a $100 \mu \mathrm{m}$ thick hydrophilic polyester film (PP2500, $3 \mathrm{M}$ ) intercalated with three layers of a $50 \mu \mathrm{m}$ thick doublesided adhesive $(467 \mathrm{MP}, 3 \mathrm{M})$. On each of these seven layers, elements of the microfluidic circuit (channels, inlets, outlets and vent 

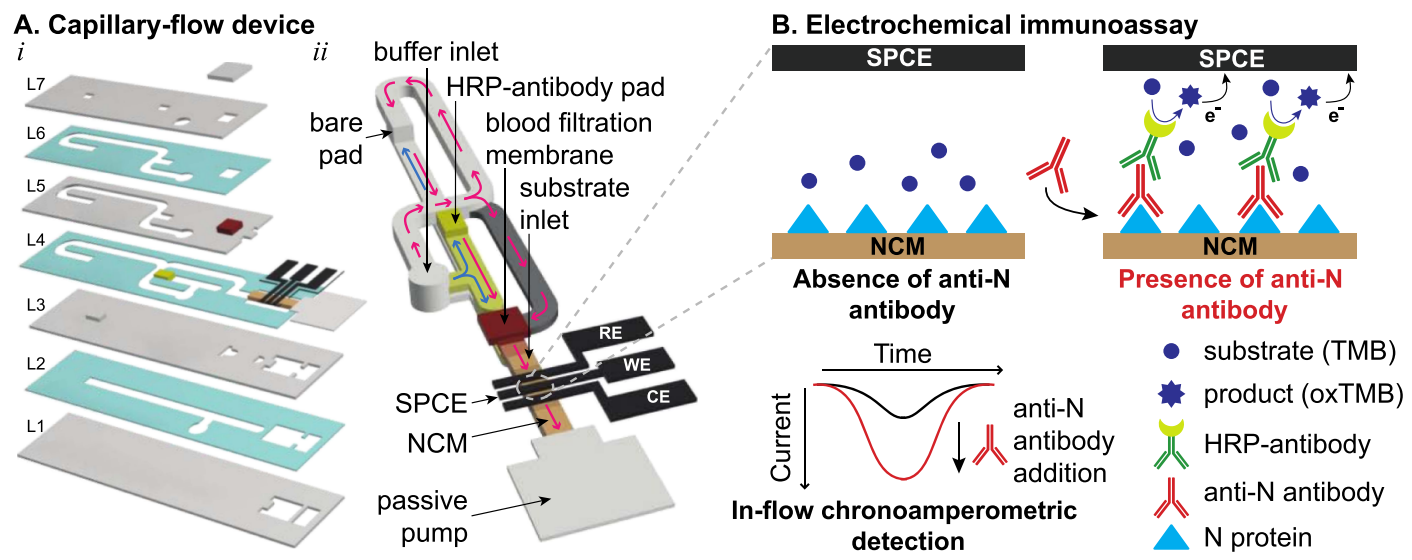

Figure 1. Schematic overview of the electrochemical capillary-flow immunoassay. (A) Exploded view (i) and 3-dimensional view (ii) of the capillary-flow device incorporating a stencil printed carbon electrode (SPCE) facing a NCM. Exploded view (i) shows the four layers of polyester film (gray, L1, L3, L5, L7) and the three layers of double-sided adhesive (turquoise, L2, L4, L6) assembled to create the 3-dimensional microfluidic network represented in (ii). Pink arrows indicate flow direction during delivery regime. In channels where flow direction is different between filling and delivery regimes, blue arrows indicate flow direction during filling regime. Where channels are superimposed, arrows indicate flow direction in both top and bottom channels. (B) Electrochemical immunoassay and detection mechanism. If present in the sample, anti-N antibodies are captured by N proteins striped on the NCM. Secondary HRP-labeled antibodies subsequently bind to anti-N antibodies and catalyze the oxidation of the substrate TMB, creating an electroactive compound (oxTMB) that is detected via chronoamperometry.

holes) were designed using CorelDRAW and cut with a $\mathrm{CO}_{2}$ laser cutter, to create the 3D microfluidic network shown in Figure 1A. An HRP-antibody reagent pad was placed in the device channel before adding the top layer of polyester film. Then, a blood-filtration membrane (Vivid GX Membrane, Pall Corporation) was placed over the sample inlet, to retain blood cells and deliver plasma to the microfluidic channel. Subsequently, a NCM striped with an N protein was inserted at the outlet of the microfluidic channel, and a waste pad (Grade 1 CHR, Whatman PLC) was placed over the end of the NCM to create a passive pump for the fluidic system. Finally, a SPCE was placed on top of the NCM, upside-down, so that the working electrode (WE) was in contact with the N protein strip. The SPCE was held in position on the NCM by the layer four adhesive (L4, Figure 1Ai).

Device Operation and Electrochemical Recording. The assay was initiated by adding a $10 \mu \mathrm{L}$ blood sample onto the blood-filtration membrane. Then, $85 \mu \mathrm{L}$ of washing buffer were added to the buffer inlet, as shown in Video S1. Once the buffer had started to flow through the NCM, a $0.0 \mathrm{~V}$ potential was applied to the WE (vs the $\mathrm{Ag} \mid \mathrm{AgCl} \mathrm{RE}$ ) using a portable potentiostat (PalmSens4) and chronoamperometry recording started. Following the sequential and automated delivery of sample, washing buffer, HRP-antibody and washing buffer to the NCM, $1 \mu \mathrm{L}$ of TMB was added to the substrate inlet on the NCM, as indicated in Figure 1Aii. In the presence of HRP in the detection zone, TMB gets oxidized into an electrochemically active product (oxTMB), which is then reduced at the electrode surface, generating an increase in the cathodic current. The electrochemical immunoassay detection mechanism is illustrated in Figure 1B.

\section{RESULTS AND DISCUSSION}

Electrochemical Capillary-Flow Device Design. The electrochemical capillary-flow device was developed with the aim of detecting anti-SARS-CoV-2 $\mathrm{N}$ protein specific antibodies with high sensitivity at the point of care. The design of the capillary-flow device was based on previous POC devices developed by our group ${ }^{32,33,37}$ and incorporated a SPCE to allow for high-sensitivity detection. Assembled layers of lasercut polyester and double-sided adhesive films created the microfluidic circuit shown in Figure 1A. This microfluidic circuit provided sequential delivery of the reagents used in the electrochemical immunoassay to a detection zone. The detection zone consisted of a NCM that was modified with a capturing agent, recombinant SARS-CoV-2 $\mathrm{N}$ protein, and a SPCE in contact with it. The SPCE had been plasma-treated for improved electrochemical performance, as previously characterized. ${ }^{41}$ The flow was maintained by an absorbent waste pad placed downstream from the detection zone.

The capillary flow-driven assay was initiated by adding a 10 $\mu \mathrm{L}$ sample of whole blood on the blood-filtration membrane. This allowed blood cells to be retained in the membrane, while plasma passed through it and entered the channel. The buffer was then added to the device, simultaneously filling all the device channels by capillary action, as illustrated by the arrows in Figure 1Aii) and shown in Video S1. This was possible, thanks to a bare glass fiber pad (L3, Figure 1Ai) and a vent hole (L7, Figure 1Ai) that were added to the buffer channel to prevent air from being trapped. The buffer flow under the blood-filtration membrane carried blood plasma to the NCM. If present in the sample, anti- $\mathrm{N}$ antibodies bound to the $\mathrm{N}$ proteins immobilized on the NCM (Figure 1B). While buffer kept flowing in the channels and through the NCM, washing away unbound sample components, HRP-antibodies were released from the reagent pad placed on L4 (Figure 1A) and subsequently delivered to the NCM. At this point, HRP antibodies bound to anti-N antibodies if present on the NCM. To prevent non-specific absorption of HRP-antibody the blood-filtration membrane, which could act as a passive pump due to evaporation, the center channel was split into two superimposed channels and a hydration channel was incorporated into the device (shown in dark gray in Figure 1Aii). This enabled the plasma to flow through the top channel while the washing buffer and the HRP-antibody flow through the bottom one (so called HRP-antibody delivery channel, shown in yellow in Figure 1Aii). Both channels converged at the end of the device, after the blood-filtration membrane but before the NCM. Evaporation of solution through the filtration membrane was also minimized by covering it with a lid made of a polyester film (Figure 1Ai). Following HRP-antibody delivery, the excess of HRP-antibody that had bound to the NCM was washed off automatically by the washing buffer running through the device. Lastly, $1 \mu \mathrm{L}$ of the TMB substrate 
was added to the substrate inlet on the NCM, as indicated in Figure 1Aii. From there, it flowed to the detection zone where it was oxidized by HRP when present. The resulting electroactive compound (oxTMB) was finally detected via chronoamperometry using the SPCE held on the NCM. The complete sandwich immunoassay and electrochemical detection mechanism are illustrated in Figure 1B.

Sequential Delivery of Reagents. The ability of the capillary-flow device to sequentially deliver reagents to the detection zone was demonstrated with a whole blood sample and color dyes to mimic reagents, as shown in Figure 2.

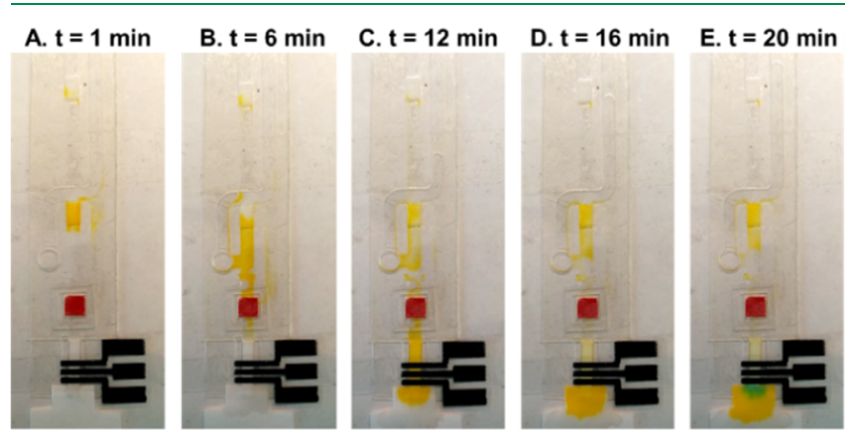

Figure 2. Sequential delivery of blood sample (A), washing buffer (B), HRP-antibodies (C) washing buffer (D), and TMB (E) to the detection area. HRP-antibody and TMB solutions are represented by yellow and blue dyes, respectively.

Once the sample and the buffer were introduced in the device, the flow started and pulled the plasma through the NCM in $1 \mathrm{~min}$ (Figure 2A). Efficient blood cell filtration through the filtration membrane was confirmed by visually assessing the color of the solution flowing through the NCM and the absorbent waste pad. While dark red residue remained on the membrane, the fluid emerging through the membrane was clear (Figure 2A). This observation is consistent with results published by our group and others. ${ }^{37,42,43}$ Following plasma delivery to the NCM and a subsequent buffer wash (Figure 2B), the yellow dye that had dried on the reagent pad to mimic the HRP-antibody was released through the HRPantibody delivery channel and flowed to the detection zone (Figure 2C, $12 \mathrm{~min}$ ). Simultaneously, buffer flowed through the hydration channel to keep the blood-filtration membrane hydrated, driving the flow to the NCM. After approximately 1 min, the HRP-antibody-mimicking yellow solution stopped flowing through the NCM and washing buffer from the buffer channel was delivered instead, to wash out excess HRPantibody (Figure $2 \mathrm{D}$ ). Lastly, $1 \mu \mathrm{L}$ of substrate-mimicking blue solution was added to the device at time $16 \mathrm{~min}$ and flowed past the detection zone in $4 \mathrm{~min}$ (Figure 2E, $20 \mathrm{~min}$ ). Overall, these results confirm that the reagents are sequentially delivered to the detection zone in less than $20 \mathrm{~min}$. Estimated incubation periods in the devices are $3 \mathrm{~min}$ for blood, $6 \mathrm{~min}$ for rehydrated HRP-antibody, and $4 \mathrm{~min}$ for TMB on the detection zone, with automatic buffer washes between them.

Static Electrochemical Detection of Anti-N Antibodies. Initial anti-N antibody assays were performed on PBSbased samples using a capillary-flow device identical to the one shown in Figure 1A, but without a blood-filtration membrane and a hydration channel (Figure S1). The first anti-N antibody measurements were carried out under static conditions. In this set up, the NCM was removed from the capillary-flow device upon completion of the fluidic assay and before addition of 1 $\mu \mathrm{L}$ of the TMB substrate. Chronoamperometry measurements started $2 \mathrm{~min}$ after the addition of TMB to the NCM. Three SPCEs were successively connected to several NCMs exposed to different anti-N antibody concentrations. Figure 3A displays
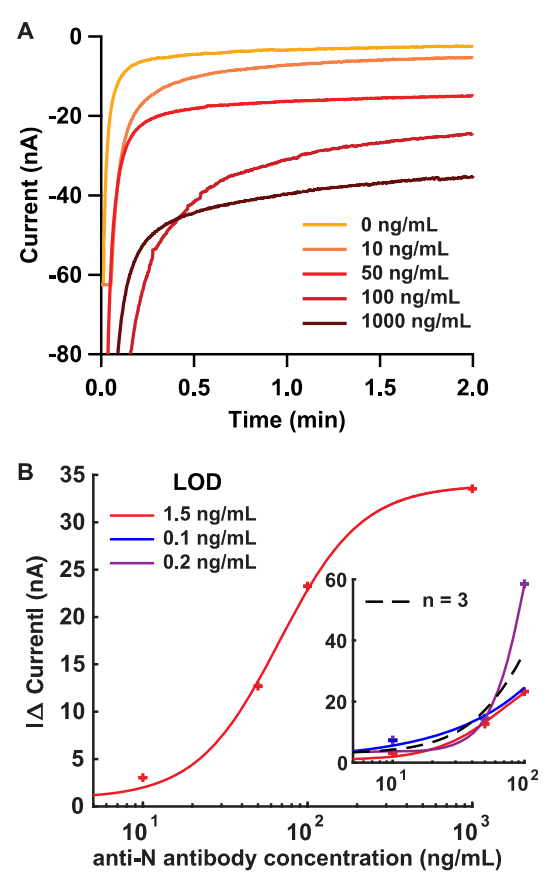

Figure 3. Static electrochemical detection of anti-N antibodies in PBS samples. (A) Chronoamperograms obtained from one SPCE consecutively connected to the NCM taken from five capillary-flow devices, each exposed to a $5 \mu \mathrm{L}$ PBS-based sample of distinct anti-N antibody concentration. (B) Corresponding calibration curve showing blank-subtracted current at $t=100 \mathrm{~s}$ (red). (Insert), amplification of $B$ showing the measurements recorded with three independent SPCEs (red, blue, and purple) and overall signal fit (black dashed line). Markers and error bars represent average and standard deviation (sd) over a $10 \mathrm{~s}$ interval centered in $100 \mathrm{~s}$. Data fitted with a 4-parameter logistic (4PL) regression. Limits of detection (LODs) are calculated as the anti-N concentration corresponding to $3 \mathrm{sd}$ of the blank signal.

the chronoamperograms recorded from a single SPCE exposed to five anti-N antibody concentrations ranging from 0 to $1 \mu \mathrm{g} /$ $\mathrm{mL}$ and the calibration curve generated by this dataset is shown in Figure 3B, together with calibration curves of the other two SPCEs (insert).

The current-to-concentration response of each SPCE followed a 4PL model, which is typical for immunoassays. ${ }^{44}$ Inherent variability of electrodes that are stencil printed by hand resulted in different LOD across the 3 SPCEs. Nevertheless, all three LODs were below $2 \mathrm{ng} / \mathrm{mL}$, demonstrating the ability of this assay to consistently detect anti-N antibody concentrations in $5 \mu \mathrm{L}$ PBS samples down to the low $\mathrm{ng} / \mathrm{mL}$ range, which outperforms commercially available $^{45,46}$ as well as recently-developed POC anti-N antibody assays. ${ }^{47-49}$ These results prove that the capillaryflow device successfully delivers sample and detection antibody to the NCM and performs effective washing steps. It also demonstrates successful electrochemical detection of TMB as the immunoassay substrate, using a SPCE placed directly on the NCM.

In-Flow Electrochemical Detection of Anti-N Antibodies. Following preliminary results in the static measure- 
ment setup, flow-based anti-N antibody tests were performed on PBS-based samples by running chronoamperometry under flow conditions. Chronoamperometry measurements were started upon delivery of buffer to the detection zone and ended upon flow completion. Three SPCEs were successively connected to four capillary-flow devices that were supplied with PBS-based samples of four different anti- $\mathrm{N}$ antibody concentrations ranging from 0 to $100 \mathrm{ng} / \mathrm{mL}$.

Under flow conditions, delivery of TMB to the detection zone produced a transient increase in the cathodic current (Figure 4A) that correlated to the concentration of anti-N

A
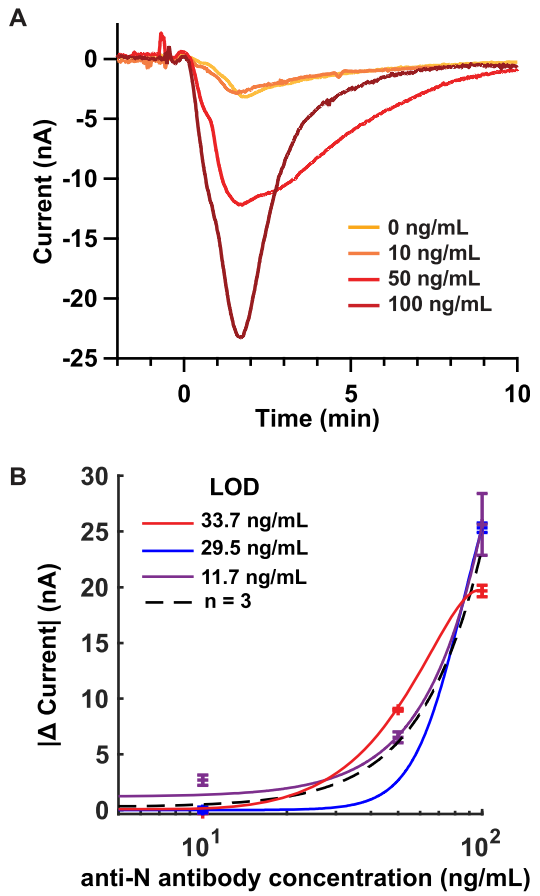

Figure 4. In-flow electrochemical detection of anti-N antibodies in PBS samples. (A) Excerpt of chronoamperograms recorded from one SPCE consecutively connected to four different capillary flow-devices, each supplied with a $5 \mu \mathrm{L}$ PBS-based sample of distinct anti-N antibody concentration. Data were time-aligned with TMB delivery to the detection zone $(t=0)$. (B) Calibration curves obtained for three independent SPCEs (red from data shown in A, blue and purple) and overall fit for all three SPCEs (black dashed line). Markers and error bars represent average and sd over a $30 \mathrm{~s}$ interval centered in the current local minimum. For each SPCE, the blank signal is subtracted from the data. Data fitted with a $4 \mathrm{PL}$ regression. LODs are calculated as the anti-N concentration corresponding to $3 \mathrm{sd}$ of the blank signal.

antibody in the sample (Figure 4B). As in the static measurement setup, the current-to-concentration response of each SPCE followed a 4PL model and variability between electrodes stencil printed by hand was observed. LODs obtained were slightly higher than those achieved in the static setup, which can be due to dispersive blurring of TMB in the microfluidic flow. However, in-flow detection of anti-N antibodies in the low $\mathrm{ng} / \mathrm{mL}$ range was still possible.

Detection of Anti-N Antibodies in Whole Human Blood. Finally, whole human blood was spiked with different anti-N antibody concentrations ranging from 0 to $100 \mathrm{ng} / \mathrm{mL}$. These samples were run on the complete fluidic device integrating the blood-filtration membrane, as shown in Figure 1 , and chronoamperometry measurements were carried out under flow conditions, as described previously. Three SPCEs were successively connected to four capillary-flow devices running these whole blood samples spiked with anti-N antibodies. In-flow chronoamperograms from one SPCE (Figure 5A) show the increase in the cathodic current
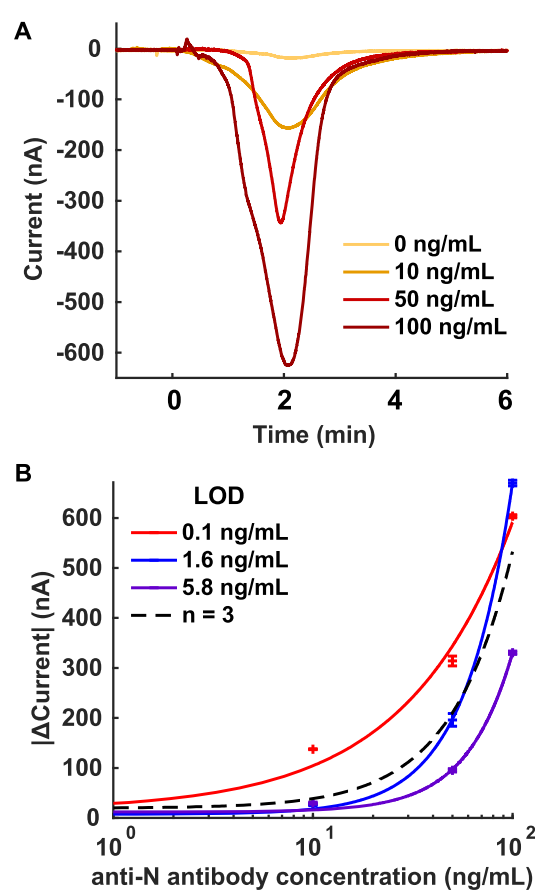

Figure 5. In-flow detection of anti-N antibodies in human whole blood samples. (A) Representative chronoamperograms recorded from one SPCE consecutively connected to four different capillary flow-devices supplied with $10 \mu \mathrm{L}$ human whole blood samples spiked with different anti-N antibody concentrations. Data were time-aligned with TMB delivery to the detection zone $(t=0)$. (B) Calibration curves recorded with three independent SPCEs (red from data shown in A, blue and purple) and overall fit for all three SPCEs (black dashed line). Markers and error bars represent average and sd over a $30 \mathrm{~s}$ interval centered in the current local minimum. For each SPCE, the blank signal is subtracted from the data. Data fitted with a 4PL regression. LODs are calculated as the anti-N concentration corresponding to $3 \mathrm{sd}$ of the blank signal.

recorded upon delivery of TMB to the detection zone. The correlation between the magnitude of this current and the concentration of anti-N antibody in the whole blood samples is shown in Figure 5B for all three SPCEs.

As for PBS-based samples, the current-to-concentration response of each SPCE followed a 4PL model and variability between electrodes was observed. This is shown by the difference in the magnitude of current response observed between the three SPCEs and highlights the variability of our current electrochemical device. The magnitude of the currents recorded here was 20 times higher than for PBS-based samples. This signal increase was attributed to three main causes: (i) blood sample volume was $10 \mu \mathrm{L}$ instead of $5 \mu \mathrm{L}$ for the PBSbased samples, to cover the entire surface of the bloodfiltration membrane and to maximize analyte extraction; (ii) delivery of blood sample to the NCM was slowed down by the blood-filtration membrane, allowing for longer sample incubation time on the detection zone, hence enhancing analyte capture; and (iii) the higher viscosity of plasma compared to PBS could further decrease the sample flowrate through the NCM. The increased signal magnitude for whole 
blood samples lead to improved analytical performance, with higher sensitivity and lower LODs down to the $5 \mathrm{ng} / \mathrm{mL}$ range (Figure 5), which matches that of commercially available tests. $^{45,46}$ Table S2 provides a side-by-side comparison of the performance of our electrochemical capillary-flow device in relation to other tests for SARS-CoV-2 anti $\mathrm{N}$ antibodies reported in the literature or commercially available. Note that our device is the only one providing a quantitative detection in whole blood samples at the point of care.

Proof-of-Concept Detection on a Smartphone. As a proof-of-concept experiment, the electrochemical capillary-flow device was coupled to an NFC potentiostat operated by a smartphone and this truly portable system was used to detect anti-N antibodies in whole blood samples. Commonly available in smartphones, NFC is a low-cost technology that enables wireless communication using a simple setup, making it ideal for use in POC applications. The NFC potentiostat (SIC4341, ISO14443A, Silicon Craft Technology PLC) was operated by an Android smartphone (Motorola One, XT1941) running a mobile application made available by Silicon Craft (SIC4341 Allstar Android app). The electrochemical capillary-flow device was connected to the NFC potentiostat via a socket mounted on the NFC potentiostat printed circuit board, in which the SPCE was inserted, as shown in Figure 6A.

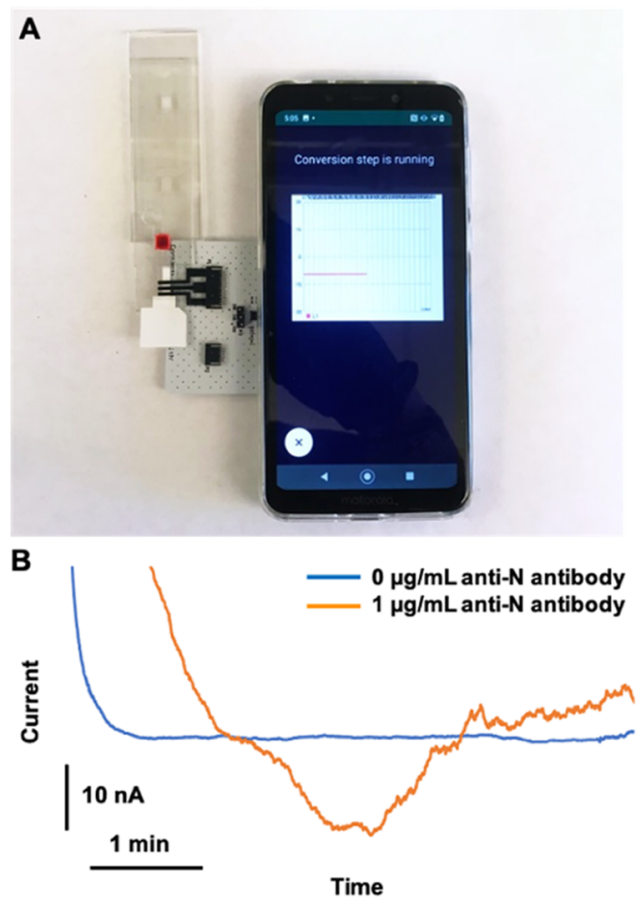

Figure 6. (A) Picture of the whole smartphone-based detection system, showing the electrochemical capillary-flow device connected to the NFC potentiostat, which is wirelessly operated from a smartphone. (B) In-flow chronoamperograms wirelessly recorded using the NFC potentiostat operated by a smartphone. Data are from two different capillary flow-devices supplied with $5 \mu \mathrm{L}$ PBS-based samples of 0 and $1 \mu \mathrm{g} / \mathrm{mL}$ anti- $\mathrm{N}$ antibody concentrations.

The connection between the NFC potentiostat and the smartphone was established by simply placing the smartphone over the NFC potentiostat, and this wireless connection automatically powered it. First, basic electrochemistry was performed to demonstrate the well-functioning of the NFC potentiostat. A cyclic voltammogram recorded from a SPCE in ferri/ferrocyanide is shown in Figure S2, and the complete measurement set up and recording is shown in Video S2. Then, to demonstrate the capability of this system to detect anti-N antibody, two electrochemical capillary-flow devices supplied with PBS-based samples of 0 (blank sample) and 1 $\mu \mathrm{g} / \mathrm{mL}$ anti-N antibody were run and chronoamperometry was performed using the NFC potentiostat. The current responses from these two measurements are displayed in Figure 6B. A clear increase in the cathodic current recorded from the $1 \mu \mathrm{g} /$ $\mathrm{mL}$ anti-N antibody sample was observed while the current from the blank sample remained constant throughout the 5 min measurement. While the concentration of anti- $\mathrm{N}$ antibody tested here was higher than the targeted clinical range, this proof-of-concept experiment demonstrates the capacity of the NFC potentiostat to be used for this assay. Further optimization of the NFC potentiostat current range is needed to allow the detection of current responses generated by samples of low anti-N antibody concentrations. This fully portable wireless smartphone-based system would greatly facilitate detection at the point of care.

\section{CONCLUSIONS}

We have presented the development of a new electrochemical capillary flow device and demonstrated its ability to detect IgG antibodies against SARS-CoV-2 $\mathrm{N}$ proteins with performance matching commercially available tests. ${ }^{45,46}$ The device is easy to use, can be fabricated at low cost $(<\$ 0.5$, see Table $S 1)$, and is single-use/disposable, making it ideal for POC applications. Thanks to an integrated blood-filtration membrane, a droplet of whole blood is added directly to the device and cells are continuously filtered, while plasma is delivered to a microfluidic circuit, which then performs the multistep assay. This eliminates the need for sample preparation, provides sequential delivery of multiple reagents to the detection zone, reduces the number of pipetting steps, and minimizes user intervention, enhancing the potential of such devices for use in a POC setting. Like in a sandwich LFIA assay, the target analyte anti$\mathrm{N}$ antibody is captured on a NCM modified with $\mathrm{N}$ protein and detected using labelled secondary antibodies. However, the device developed here uses electrochemical detection via a SPCE that is placed on the NCM and connected to a portable potentiostat. While maintaining the ease of use and the rapidity of a LFIA, this novel system provides a highly sensitive detection, with detection limits lower than $10 \mathrm{ng} / \mathrm{mL}$ anti-N antibody in human whole blood samples as small as $10 \mu \mathrm{L}$. Critically, the electrochemical detection provides a quantitative output, eliminating any possible user interpretation error, which is a major advantage of the system to be used at the point of care. In a proof-of-concept experiment, the device was successfully used with a low-cost NFC potentiostat operated from a smartphone. This potentiostat could be integrated with the device in the future, so that the whole system is disposable. This would eliminate the need for any dedicated instrumentation as the test could be run from a smartphone only, making it useable at the point of need. Future work shall focus on tuning the NFC potentiostat to match its recording range to output signals from samples of clinically low analyte concentration. Whilst there is preliminary evidence showing stability over storage of every active component of the device (the electrode, ${ }^{41}$ the $\mathrm{N}$ protein modified $\mathrm{NCM}^{37}$ and the detection antibody reagent pad $^{40}$ ), a stability study of the complete device was not conducted yet and would be necessary for the device to access the POC market. Lastly, further developments 
are needed to increase the reproducibility of our measurements. We believe that variability between electrodes, which are currently stencil-printed by hand, could be eliminated by screen-printing electrodes in batches, as it is performed in manufacturing settings. Additionally, manufacturing capillaryflow devices in batches would allow for uniform assembly of the device layers and provide a consistent pressure between the electrodes and the NCM. This is difficult to accomplish by hand assembly but is necessary to enhance the reproducibility of our quantitative detection to translate it to real-world POC use. Applied to the detection of other pathogens, this novel electrochemical capillary-flow technology has the potential to aid the diagnosis of multiple infectious diseases at the point of care.

\section{ASSOCIATED CONTENT}

\section{SI Supporting Information}

The Supporting Information is available free of charge at https://pubs.acs.org/doi/10.1021/acssensors.1c01527.

Schematics of capillary-flow device without bloodfiltration membrane, cyclic voltammogram recorded with NFC potentiostat, and cost of capillary-flow device (PDF)

$32 \mathrm{x}$-accelerated video of capillary-flow device operation (MP4)

Video of NFC potentiostat operation (MP4)

\section{AUTHOR INFORMATION}

\section{Corresponding Author}

Charles S. Henry - Department of Chemistry, Department of Chemical and Biological Engineering, and School of Biomedical Engineering, Colorado State University, Fort Collins, Colorado 80523, United States; (1) orcid.org/00000002-8671-7728; Email: chuck.henry@colostate.edu

\section{Authors}

Isabelle C. Samper - Department of Chemistry and Department of Chemical and Biological Engineering, Colorado State University, Fort Collins, Colorado 80523, United States

Ana Sánchez-Cano - Department of Chemistry, Colorado State University, Fort Collins, Colorado 80523, United States; Diagnostic Nanotools Group, Institut de Recerca, Barcelona 08035, Spain; Universitat Autónoma de Barcelona, Barcelona 08193, Spain

Wisarut Khamcharoen - Department of Chemistry, Colorado State University, Fort Collins, Colorado 80523, United States; Department of Chemistry, Faculty of Science, Srinakharinwirot University, Bangkok 10110, Thailand

Ilhoon Jang - Department of Chemistry, Colorado State University, Fort Collins, Colorado 80523, United States; Institute of Nano Science and Technology, Hanyang University, Seoul 04763, South Korea

Weena Siangproh - Department of Chemistry, Faculty of Science, Srinakharinwirot University, Bangkok 10110, Thailand

Eva Baldrich - Diagnostic Nanotools Group, Institut de Recerca, Barcelona 08035, Spain; 이이.org/0000-00031393-215X

Brian J. Geiss - Department of Microbiology, Immunology, and Pathology and School of Biomedical Engineering,
Colorado State University, Fort Collins, Colorado 80523, United States

David S. Dandy - Department of Chemical and Biological Engineering and School of Biomedical Engineering, Colorado State University, Fort Collins, Colorado 80523, United States; (1) orcid.org/0000-0003-3254-0717

Complete contact information is available at:

https://pubs.acs.org/10.1021/acssensors.1c01527

\section{Author Contributions}

I.C.S., A.S.-C. and W.K. contributed equally.

Notes

The authors declare no competing financial interest.

\section{ACKNOWLEDGMENTS}

We would like to thank James Terry and Loran Anderson for providing recombinant SARS-CoV-2 $\mathrm{N}$ protein. This work has been supported by the NIH (R01 AI132668), the Insituto de Salud Carlos III and the European regional Development Fund (IFI18/00020, MV19/00036, CPII18/00025), the Science Achievement Scholarship of Thailand (SAST), the Colorado Office of Economic Development and International Trade (OEDIT), and Balanced Biotech Inc.

\section{REFERENCES}

(1) WHO. WHO Coronavirus (COVID-19) DashboardIWHO Coronavirus Disease (COVID-19) Dashboard. https://covid19.who. int/ (accessed Jul 10, 2021).

(2) IHE. Testing, Screening, and Outbreak Response for Institutions of Higher Education (IHEs)ICDC. https://www.cdc.gov/ coronavirus/2019-ncov/community/colleges-universities/ihe-testing. html (accessed Mar 12, 2021).

(3) Pollán, M.; Pérez-Gómez, B.; Pastor-Barriuso, R.; Oteo, J.; Hernán, M. A.; Pérez-Olmeda, M.; Sanmartín, J. L.; FernándezGarcía, A.; Cruz, I.; de Larrea, N. F.; et al. Prevalence of SARS-CoV-2 in Spain (ENE-COVID): A Nationwide, Population-Based Seroepidemiological Study. Lancet 2020, 396, 535-544.

(4) Nuccetelli, M.; Pieri, M.; Grelli, S.; Ciotti, M.; Miano, R.; Andreoni, M.; Bernardini, S. SARS-CoV-2 Infection Serology: A Useful Tool to Overcome Lockdown? Cell Death Discovery 2020, 6, $1-9$.

(5) Böger, B.; Fachi, M. M.; Vilhena, R. O.; Cobre, A. F.; Tonin, F. S.; Pontarolo, R. Systematic Review with Meta-Analysis of the Accuracy of Diagnostic Tests for COVID-19. Am. J. Infect. Control 2021, 49, 21-29.

(6) Kevadiya, B. D.; Machhi, J.; Herskovitz, J.; Oleynikov, M. D.; Blomberg, W. R.; Bajwa, N.; Soni, D.; Das, S.; Hasan, M.; Patel, M.; et al. Diagnostics for SARS-CoV-2 Infections. Nat. Mater. 2021, 20, 593-605.

(7) Salazar, E.; Kuchipudi, S. V.; Christensen, P. A.; Eagar, T.; Yi, X.; Zhao, P.; Jin, Z.; Long, S. W.; Olsen, R. J.; Chen, J.; et al. Convalescent Plasma Anti-SARS-CoV-2 Spike Protein Ectodomain and Receptor-Binding Domain IgG Correlate with Virus Neutralization. J. Clin. Invest. 2020, 130, 6728-6738.

(8) Kopel, J.; Goyal, H.; Perisetti, A. Antibody Tests for COVID-19. Proc.-Bayl. Univ. Med. Cent. 2021, 34, 63-72.

(9) Emanuel, E. J.; Persad, G.; Upshur, R.; Thome, B.; Parker, M.; Glickman, A.; Zhang, C.; Boyle, C.; Smith, M.; Phillips, J. P. Fair Allocation of Scarce Medical Resources in the Time of Covid-19. N. Engl. J. Med. 2020, 382, 2049-2055.

(10) Sheridan, C. Fast, Portable Tests Come Online to Curb Coronavirus Pandemic. Nat. Biotechnol. 2020, 38, 515-518.

(11) Patel, E. U.; Bloch, E. M.; Clarke, W.; Hsieh, Y. H.; Boon, D.; Eby, Y.; Fernandez, R. E.; Baker, O. R.; Keruly, M.; Kirby, C. S.; et al. Comparative Performance of Five Commercially Available Serologic 
Assays to Detect Antibodies to SARS-CoV-2 and Identify Individuals with High Neutralizing Titers. J. Clin. Microbiol. 2021, 59, 1-10.

(12) Charlton, C. L.; Kanji, J. N.; Johal, K.; Bailey, A.; Plitt, S. S.; MacDonald, C.; Kunst, A.; Buss, E.; Burnes, L. E.; Fonseca, K.; et al. Evaluation of Six Commercial Mid- To High-Volume Antibody and Six Point-of-Care Lateral Flow Assays for Detection of SARS-CoV-2 Antibodies. J. Clin. Microbiol. 2020, 58, 1-12.

(13) Andryukov, B. G. Six Decades of Lateral Flow Immunoassay: From Determining Metabolic Markers to Diagnosing Covid-19. AIMS Microbiol. 2020, 6, 280-304.

(14) Liu, Y.; Zhan, L.; Qin, Z.; Sackrison, J.; Bischof, J. C. Ultrasensitive and Highly Specific Lateral Flow Assays for Point-ofCare Diagnosis. ACS Nano 2021, 15, 3593-3611.

(15) Cesewski, E.; Johnson, B. N. Electrochemical Biosensors for Pathogen Detection. Biosens. Bioelectron. 2020, 159, 112214.

(16) Monzó, J.; Insua, I.; Fernandez-Trillo, F.; Rodriguez, P. Fundamentals, Achievements and Challenges in the Electrochemical Sensing of Pathogens. Analyst 2015, 140, 7116-7128.

(17) Xu, X.; Zhang, S.; Chen, H.; Kong, J. Integration of Electrochemistry in Micro-Total Analysis Systems for Biochemical Assays: Recent Developments. Talanta 2009, 80, 8-18.

(18) de Eguilaz, M. R.; Cumba, L. R.; Forster, R. J. Electrochemical Detection of Viruses and Antibodies: A Mini Review. Electrochem. Commun. 2020, 116, 106762.

(19) Li, X.-M.; Yang, X.-Y.; Zhang, S.-S. Electrochemical Enzyme Immunoassay Using Model Labels. Trac. Trends Anal. Chem. 2008, 27, 543-553.

(20) Layqah, L. A.; Eissa, S. An Electrochemical Immunosensor for the Corona Virus Associated with the Middle East Respiratory Syndrome Using an Array of Gold Nanoparticle-Modified Carbon Electrodes. Microchim. Acta 2019, 186, 1-10.

(21) Ozer, T.; Geiss, B. J.; Henry, C. S. Chemical and Biological Sensors for Viral Detection. J. Electrochem. Soc. 2020, 167, 037523.

(22) Zhao, C.; Liu, X. A Portable Paper-Based Microfluidic Platform for Multiplexed Electrochemical Detection of Human Immunodeficiency Virus and Hepatitis C Virus Antibodies in Serum. Biomicrofluidics 2016, 10, 024119.

(23) Wang, L.; Filer, J. E.; Lorenz, M. M.; Henry, C. S.; Dandy, D. S.; Geiss, B. J. An Ultra-Sensitive Capacitive Microwire Sensor for Pathogen-Specific Serum Antibody Responses. Biosens. Bioelectron. 2019, 131, 46-52.

(24) da Silva, E. T. S. G.; Souto, D. E. P.; Barragan, J. T. C.; de Giarola, J. F.; de Moraes, A. C. M.; Kubota, L. T. Electrochemical Biosensors in Point-of-Care Devices: Recent Advances and Future Trends. ChemElectroChem 2017, 4, 778-794.

(25) Ruiz-Vega, G.; Arias-Alpízar, K.; de la Serna, E.; BorghetiCardoso, L. N.; Sulleiro, E.; Molina, I.; Fernàndez-Busquets, X.; Sánchez-Montalvá, A.; del Campo, F. J.; Baldrich, E. Electrochemical POC Device for Fast Malaria Quantitative Diagnosis in Whole Blood by Using Magnetic Beads, Poly-HRP and Microfluidic Paper Electrodes. Biosens. Bioelectron. 2020, 150, 111925.

(26) Sun, A. C.; Hall, D. A. Point-of-Care Smartphone-Based Electrochemical Biosensing. Electroanalysis 2019, 31, 2-16.

(27) Wan, Y.; Su, Y.; Zhu, X.; Liu, G.; Fan, C. Development of Electrochemical Immunosensors towards Point of Care Diagnostics. Biosens. Bioelectron. 2013, 47, 1-11.

(28) Samper, I. C.; Gowers, S. A. N.; Booth, M. A.; Wang, C.; Watts, T.; Phairatana, T.; Vallant, N.; Sandhu, B.; Papalois, V.; Boutelle, M. G. Portable Microfluidic Biosensing System for Real-Time Analysis of Microdialysate in Transplant Kidneys. Anal. Chem. 2019, 91, 1463114638 .

(29) Mettakoonpitak, J.; Boehle, K.; Nantaphol, S.; Teengam, P.; Adkins, J. A.; Srisa-Art, M.; Henry, C. S. Electrochemistry on PaperBased Analytical Devices: A Review. Electroanalysis 2016, 28, 14201436.

(30) Noviana, E.; McCord, C. P.; Clark, K. M.; Jang, I.; Henry, C. S. Electrochemical Paper-Based Devices: Sensing Approaches and Progress toward Practical Applications. Lab Chip 2020, 20, 9-34.
(31) Ataide, V. N.; Mendes, L. F.; Gama, L. I. L. M.; de Araujo, W. R.; Paixão, T. R. L. C. Electrochemical Paper-Based Analytical Devices: Ten Years of Development. Anal. Methods 2020, 12, 10301054.

(32) Jang, I.; Carrão, D. B.; Menger, R. F.; Moraes De Oliveira, A. R.; Henry, C. S. Pump-Free Microfluidic Rapid Mixer Combined with a Paper-Based Channel. ACS Sens. 2020, 5, 2230-2238.

(33) Jang, I.; Kang, H.; Song, S.; Dandy, D. S.; Geiss, B. J.; Henry, C. $\mathrm{S}$. Flow Control in a Laminate Capillary-Driven Microfluidic Device. Analyst 2021, 146, 1932-1939.

(34) Burbelo, P. D.; Riedo, F. X.; Morishima, C.; Rawlings, S.; Smith, D.; Das, S.; Strich, J. R.; Chertow, D. S.; Davey, R. T.; Cohen, J. I. Sensitivity in Detection of Antibodies to Nucleocapsid and Spike Proteins of Severe Acute Respiratory Syndrome Coronavirus 2 in Patients With Coronavirus Disease 2019. J. Infect. Dis. 2020, 222, 206-213.

(35) Azkur, A. K.; Akdis, M.; Azkur, D.; Sokolowska, M.; Veen, W.; Brüggen, M. C.; O’Mahony, L.; Gao, Y.; Nadeau, K.; Akdis, C. A. Immune Response to SARS-CoV-2 and Mechanisms of Immunopathological Changes in COVID-19. Allergy 2020, 75, 1564-1581.

(36) Batra, M.; Tian, R.; Zhang, C.; Clarence, E.; Sacher, C. S.; Miranda, J. N.; De La Fuente, J. R. O.; Mathew, M.; Green, D.; Patel, S.; et al. Role of IgG against N-Protein of SARS-CoV2 in COVID19 Clinical Outcomes. Sci. Rep. 2021, 11, 3455.

(37) Carrell, C.; Link, J.; Jang, I.; Terry, J.; Scherman, M.; Call, Z.; Panraksa, Y.; Dandy, D. S.; Geiss, B. J.; Henry, C. Point-of-Need Disposable ELISA System for COVID-19 Serology Testing. ChemRxiv 2020, DOI: $10.26434 /$ chemrxiv.13050539.v2.

(38) Fagre, A.; Lewis, J.; Eckley, M.; Zhan, S.; Rocha, S. M.; Sexton, N. R.; Burke, B.; Geiss, B.; Peersen, O.; Bass, T.; et al. SARS-CoV-2 Infection, Neuropathogenesis and Transmission among Deer Mice: Implications for Spillback to New World Rodents. PLoS Pathog. 2021, 17, No. e1009585.

(39) Terry, J. S.; Anderson, L. B.; Scherman, M. S.; McAlister, C. E.; Perera, R.; Schountz, T.; Geiss, B. J. Development of a SARS-CoV-2 Nucleocapsid Specific Monoclonal Antibody. Virology 2021, 558, 2837.

(40) Ramachandran, S.; Fu, E.; Lutz, B.; Yager, P. Long-Term Dry Storage of an Enzyme-Based Reagent System for ELISA in Point-ofCare Devices. Analyst 2014, 139, 1456-1462.

(41) Kava, A. A.; Henry, C. S. Exploring Carbon Particle Type and Plasma Treatment to Improve Electrochemical Properties of StencilPrinted Carbon Electrodes. Talanta 2021, 221, 121553.

(42) Liu, C.; Liao, S.-C.; Song, J.; Mauk, M. G.; Li, X.; Wu, G.; Ge, D.; Greenberg, R. M.; Yang, S.; Bau, H. H. A High-Efficiency Superhydrophobic Plasma Separator. Lab Chip 2016, 16, 553-560.

(43) Su, X.; Zhang, J.; Zhang, D.; Wang, Y.; Chen, M.; Weng, Z.; Wang, J.; Zeng, J.; Zhang, Y.; Zhang, S.; et al. High-Efficiency Plasma Separator Based on Immunocapture and Filtration. Micromachines 2020, 11, 352.

(44) O’Connell, M. A.; Belanger, B. A.; Haaland, P. D. Calibration and Assay Development Using the Four-Parameter Logistic Model. Chemom. Intell. Lab. Syst. 1993, 20, 97-114.

(45) GmbH. ABIN6952772 SARS-CoV-2 N-Protein IgG Antibody (SARS-CoV-2 N IgG) ELISA Kit. https://www.antibodies-online. com/productsheets/ABIN6952772.pdf (accessed May 11, 2021).

(46) Protein Tech. Anti-SARS-CoV-2 N protein Human IgG ELISA Kit Elisa Kit KE30001 Proteintech. https://www.ptglab.com/ Products/Pictures/pdf/Virus-IgG-for-2019-nCoV-N-protein-ELISAKit-KE30001-datasheet.pdf (accessed Mar 18, 2021).

(47) Kim, S.; Hao, Y.; Miller, E. A.; Tay, D. M. Y.; Yee, E.; Kongsuphol, P.; Jia, H.; McBee, M.; Preiser, P. R.; Sikes, H. D. Vertical Flow Cellulose-Based Assays for SARS-CoV-2 Antibody Detection in Human Serum. ACS Sens. 2021, 6, 1891-1898.

(48) Kasetsirikul, S.; Umer, M.; Soda, N.; Sreejith, K. R.; Shiddiky, M. J. A.; Nguyen, N.-T. Detection of the SARS-CoV-2 Humanized Antibody with Paper-Based ELISA. Analyst 2020, 145, 7680-7686.

(49) Wang, Z.; Zheng, Z.; Hu, H.; Zhou, Q.; Liu, W.; Li, X.; Liu, Z.; Wang, Y.; Ma, Y. A Point-of-Care Selenium Nanoparticle-Based Test 
for the Combined Detection of Anti-SARS-CoV-2 IgM and IgG in

Human Serum and Blood. Lab Chip 2020, 20, 4255-4261. 